\title{
LA CERÁMICA DE BARNIZ NEGRO ITÁLICA EN EL TERRITORIUM DE LA ANTIGUA CIUDAD ROMANA DE CONSABVRA (CONSUEGRA, TOLEDO)
}

\author{
BLACK GLAZED CERAMIC IN THE TERRITORY OF ANCIENT ROMAN TOWN OF CONSABVRA \\ (CONSUEGRA, TOLEDO)
}

\author{
JUAN FRANCISCO PALENCIA GARCÍA \\ DIEGO RODRÍGUEZ LÓPEZ-CANO \\ Universidad Nacional de Educación a Distancia
}

\section{INTRODUCCIÓN}

El presente trabajo pretende empezar a dar a conocer una de las ciudades carpetanas menos estudiadas en su desarrollo histórico durante la Antigüedad, de hecho es la menos conocida de las tres grandes ciudades carpetanas que tras el periodo de conquista, en torno a mediados del s. II a.C., pasarían a integrarse primero en la Citerior y posteriormente en el área NW del conventus Carthaginensis, dentro de la Tarraconense y que actuaron como cabeceras administrativas, junto a Toletum y Complutum (Plin. Hist. Nat., III, 25). Entre las razones de su abandono historiográfico destaca su ubicación en un núcleo de fuerte tradición rural, Consuegra, que se encuentra al SE de la provincia de Toledo, en la comarca de la Mancha Alta.

Por otra parte, muchos de los restos arqueológicos se sitúan bajo la actual estructura urbana ${ }^{1}$, con la problemática que de ello se deriva para su estudio (Palencia, 2011, 129-177).

Pese a estas dificultades, sabemos con certeza que el oppidum prerromano ubicado en el Cerro Calderico $(828 \mathrm{~m})$ daría paso a una gran ciudad romana con su traslado ad planum, junto al cauce del río Amarguillo. Prueba de ello, son los restos conservados: presa, estanques, tramos de specus, circo, estatuas forales, inscripciones, etc. (Aguirre, 1769). Pero tenemos la certeza de que este traslado fue progresivo y lento, como demuestran los restos materiales aparecidos hasta el momento en dicho cerro, muchos de los cuales son de época tardorrepublicana y altoimperial.

\footnotetext{
1. Los informes arqueológicos urbanos comenzaron a realizarse a partir del 2007. Archivo del Museo de Santa Cruz de Toledo (A.M.S.C.T.). Queremos agradecer tanto a los arqueólogos que trabajaron tanto en la Carta Arqueológica como en los citados informes urbanos las facilidades que nos dieron para la consulta de estos materiales. Por otra parte, queremos extender estos agradecimientos a los responsables del $\mathrm{Mu}-$ seo Arqueológico Municipal de Consuegra, Museo de Santa Cruz de Toledo y Museo Provincial de Ciudad Real.
}

Por último, este artículo pretende dar una visión de conjunto sobre la cerámica Campaniense o mejor dicho, de barniz negro itálica, aparecida hasta el momento en la zona. La importancia de este tipo de cerámica tardorrepublicana radica en que se asocia a la conquista de Hispania por Roma, y en concreto al inicio del proceso de romanización de la Meseta Sur. La fecha de los años 50/40 a.C. significa el final de las exportaciones de la Campaniense hacia Hispania, estando por completo ausente de los niveles augusteos (Beltrán, 1990, 39-40; Principal y Ribera, 2013, 43).

\section{ETAPA REPUBLICANA: CIUDAD Y TERRITORIO}

\section{LA CIVITAS DE CONSABVRA}

Durante esta época se produce la perduración del oppidum carpetano en el Cerro Calderico, al menos hasta las guerras sertorianas, es decir, el primer cuarto del s. I a.C., este hecho se confirma a partir de dos tipos de fuentes históricas:

A) Las literarias grecolatinas, muy importantes para el análisis del proceso de la conquista romana de la Meseta Sur, que terminaría para nuestra zona de estudio a mediados del s. II a.C. (Carrasco, 2008, 27). La fuente literaria en cuestión es escueta, aunque interesante (PS. FRONTIN. Strat. IV, 5, 19²), ya que de la

2. PS. FRONTIN., Strat. IV, 5,19. Se hace mención por primera vez al oppidum de Consabura: Hispani Consabrae obsessi eadem omnia passi sunt nec oppidum Hirtuleio tradiderunt: «Los hispanos de Consabura han soportado estas mismas cosas y asediados no entregaron el oppidum a Hirtuleyo». Sexto Julio Frontino, que fue cónsul en dos ocasiones, escribió en la segunda mitad del siglo I d.C. una famosa obra sobre la conducción de aguas a Roma (De aquis urbis Romae), considerada como uno de los mejores tratados técnicos en latín, y tres libros de estratagemas militares (Stratagemae). 
misma se pueden deducir una serie de aspectos como la resistencia de Consabura a la revuelta de Sertorio, siendo por tanto «fiel a la administración romana» (a las tropas de M. Domicio Calvino propretor de la Citerior), y por tanto, resistiendo el asedio del cuestor de Sertorio L. Hirtuleyo en el año 79-78 a.C.

B) Los materiales arqueológicos encontrados en el Cerro Calderico (colecciones del Museo Municipal de Consuegra y Museo de Santa Cruz de Toledo ${ }^{3}$ ): las cerámicas de barniz negro o Campanienses ${ }^{4}$, los glandes o proyectiles de plomo, la numismática (denarios de la ceca Bolskan ${ }^{5}$ )... todos ellos nos trasladan a la etapa tardorrepublicana en la Meseta Sur.

\section{SU VASTO Y ESTRATÉGICO TERRITORIUM}

En este periodo se produce una reorganización del vasto territorio de la ciudad, debido a su posición estratégica (entre los valles del Guadiana y del Tajo) y a sus ricos recursos naturales: por una parte, los agropecuarios, sobre todo por sus importantes áreas de pastos en torno a las lagunas salinas del sector este de la ciudad, lagunas de los actuales términos municipales de la provincia de Toledo y Ciudad Real: Villacañas, Lillo, Quero, Villafranca de los Caballeros, Alcázar de San Juan (Muñoz, 2008, 527-556), y a los territorios colindantes a la presa romana situada en la vega del río Amarguillo dedicados a las actividades agrícolas; por otro lado, tenemos la certeza de una actividad minera, en concreto, minas de cobre y de galena argentífera, en la parte sur y oeste de la ciudad (Montero, Rodríguez y Rojas, 1990, 18, 23-24). De este modo, nos llama la atención la concentración de

Existe un cuarto libro de Estratagemas, que al parecer no se debe a su autoría, a su autor por ello se le conoce con el nombre del Pseudo Frontino.

3. Agradecemos a los responsables de ambos museos, las facilidades a la hora de acceder y estudiar estos materiales.

4. Salvo la asita de una tapa de un vaso de cerámica Campaniense $\mathrm{C}$, hallada en las excavaciones del circo romano (Giles Pacheco, 1971, 153). El resto de los fragmentos (cinco), fueron hallados en la ladera noreste del Cerro Calderico. Datos corroborados en el inventario de las colecciones del Museo Municipal de Consuegra, realizado por FernándezLayos, durante los años 80 del siglo XX. Números de Inventario: 428-33 (61-63), 473 (64) y 474 (65). Al menos uno de los fragmentos es de Campaniense A: 474 (65), en concreto del de producción del Golfo de Nápoles, s. III- I a.C. Esta idea fue corroborada por el especialista en cerámica romana D. Albert Ribera i Lacomba, arqueólogo del Ayuntamiento de Valencia, al que tuvimos ocasión de conocer en el Curso de cerámica romana: «La cerámica romana una valiosa herramienta de la historia». Museo Arqueológico Regional (MAR). Alcalá de Henares. Octubre-Noviembre, 2010.

5. Hemos documentado un ejemplar en los Fondos del Museo Municipal de Consuegra, junto a otro procedente de una colección particular. Ambos aparecieron en el Cerro Calderico. minas cercanas a la vía 30 del Itinerario de Antoni$n^{6}$, en el llamado antiguo camino Consuegra-Puerto Lápice, al SE del actual núcleo urbano (Palencia y Rodríguez, 2013, 58-71).

Como mencionábamos, creemos que tras ser sofocada la rebelión de Sertorio (82-72 a.C.), la administración romana impuso una fuerte reestructuración del territorio de la zona (esto fue algo habitual por parte de Roma a lo largo de la República, lo observamos con Graco $^{7}$ o con César $\left.{ }^{8}\right)$. Buenos ejemplos de ciudades abandonadas de la Meseta Sur durante esta época son los yacimientos de Plaza de Moros (Villatobas, Toledo), destruida por el fuego en el siglo II a.C.; Fosos de Bayona (Villas Viejas, Cuenca), ¿quizás en beneficio de Segobriga?, ocupada hasta el primer tercio del s. I a.C.; La Muela de Alcocer (Guadalajara) cuya población se traslada hacia El Castro de Santaver, en el caso de Ercavica; y Alarcos (Ciudad Real), abandonada a finales del s. I a.C. (Curchin, 2012, 16).

De esta manera, comprobamos como en nuestra zona de estudio algunos centros de población son abandonados tras la revuelta sertoriana, mientras que otros simplemente se trasladan, hacia un emplazamiento, generalmente cercano. Sirva como ejemplo de lo primero el caso del oppidum coetáneo de El Gollino en Corral de Almaguer (Toledo), cuya cronología llega hasta el siglo I a.C., tras los escarceos de Sertorio por estas tierras (Santos, Perea y Prados, 1990, 309-325); mientras que para los traslados poblacionales, el caso del comentado Cerro Calderico de Consuegra, es el más significativo.

Cuando no contamos con epigrafía disponible para trazar los límites de una ciudad romana, contamos con otras técnicas dentro de la arqueología del paisaje, tales como la observación de accidentes geográficos que pudieron haber tenido una función delimitadora: ríos, montes y sierras (Schattner, Ovejero y Pérez, 2008, 131), incluso caminos (Rodríguez Morales, 2007, 34).

6. La vía XXX del Itinerarium Antonini: es una de las vías más cortas con 95 millas distancia, el equivalente a algo más de $133 \mathrm{~km}$. Consabro en el Itinerario de Antonino $(446,6)$ y Consabron en el Anónimo de Rávena $(313,15)$.

7. Según Estrabón, III, 4, 13: «cuando Polibio dice que Graco ha destruido trescientas ciudades, sonríe (Poseidonio) pues llama ciudades a simples fortines». Según Floro (I, 33, 9), Graco castigó a los celtíberos al demoler ciento cincuenta ciudades, fundó Gracurris (Alfaro, La Rioja), (LIV., Per., 41) y obtuvo la sumisión de los celtíberos. Por tanto, pasó de la Carpetania a la Celtiberia, donde las numerosas victorias conseguidas en la región provocaron el sometimiento voluntario de la ciudad de Ercavica (Cañaveruelas, al NW de la provincia de Cuenca).

8. Dion Casio (XL, 39, 5), nos recuerda los favores que César otorgó a las poblaciones después de la batalla de Munda (45 a.C.): «En cuanto aquellos de quienes había recibido cualquier tipo de apoyo, a unos concedió tierras y los eximió de impuestos, a otros otorgó la ciudadanía y a otros el rango de colonos romanos; no obstante, no hacía tales favores a cambio de nada». 
Una forma de comenzar puede ser la aplicación de la técnica de los polígonos Thiessen: «se dibujan polígonos en torno a las ciudades, trazando simplemente unas perpendiculares por los puntos medios entre las ciudades» (Hodder y Orton, 1990, 72). Esta técnica se basa en la premisa de que es más probable que un asentamiento interactúe con la ciudad más cercana, y ha sido aplicada en numerosos ejemplos.

En la figura 1 se muestra un mapa de la zona de nuestro estudio; si partimos de la base de considerar sólo los núcleos romanos más importantes a la hora de establecer los correspondientes polígonos Thiessen, marcaríamos en el mapa inicial la localización de las ciudades de Consabura (Consuegra), Toletum (Toledo), Segobriga (Saelices, Cuenca) y Laminium (Alhambra, Ciudad Real), entre otras.

Pero además creemos, que a la hora de fijar los límites del territorio consaburense estos estarían delimitados por accidentes geográficos naturales, siguiendo lo que los antiguos agrimensores (gromatici) denominaban: «ager per extremitatem mensura comprehensus» (Front. Th. 1,2), propio de las ciudades estipendiarias como Consabura. Prueba de esta hipótesis es el yacimiento excavado de Pozos de Finisterre (Hernández y Morín de Pablos, 2008, 45-49), situado a unos 20 km al norte de la actual Consuegra, en la margen derecha del río Algodor, cuyo origen es tardorrepublicano, a tenor de los materiales arqueológicos encontrados. $\mathrm{La}$ importancia de este yacimiento, que no es una villa, sino una posible statio, estriba por un lado en el descubrimiento de restos de calzada, pudiéndose relacionar con la vía 30 del Itinerario de Antonino (Fernández, Seldas y Zarzalejos, 1990, 165-182).

Por otra parte, la toponimia del lugar es muy interesante, ya que nos está pudiendo informar sobre el límite del territorium de Consabura al N. El nombre Finisterre, parece relacionarse con el vocablo latino fines-ium, límites o fronteras, cuyo ablativo plural sería finibus, que se asemeja al antiguo vocablo medieval de Finibusterre (Aguirre, 1769, 137). Aquí Finibus terrae, no tiene el sentido del conocido «fin del mundo o de la tierra», sino el fin de un territorium de una ciuitas y el comienzo de otro, es decir, el fin de la jurisdicción territorial de Consabura y el inicio de la de Toletum.

Otro elemento que podría servir para delimitar el territorio de la ciudad, en este caso en su parte este, creemos que sería la propia vía 29 del Itinerario de Antonino (Per Lusitaniam ab Emerita Caesarea Augus$t a)$. Sabemos por las fuentes grecolatinas que una vez conquistado un territorio, una comisión de expertos, entre los que había siempre los citados agrimensores (gromatici), determinaba los límites de una ciudad y para los mismos se fijaban tanto en los mencionados límites naturales (basados en ocasiones en delimitaciones indígenas previas), como el paso de una vía (Front., Gromat., Th., II, 10-11).

De esta forma, una hipótesis de los límites de este territorium podría ser:

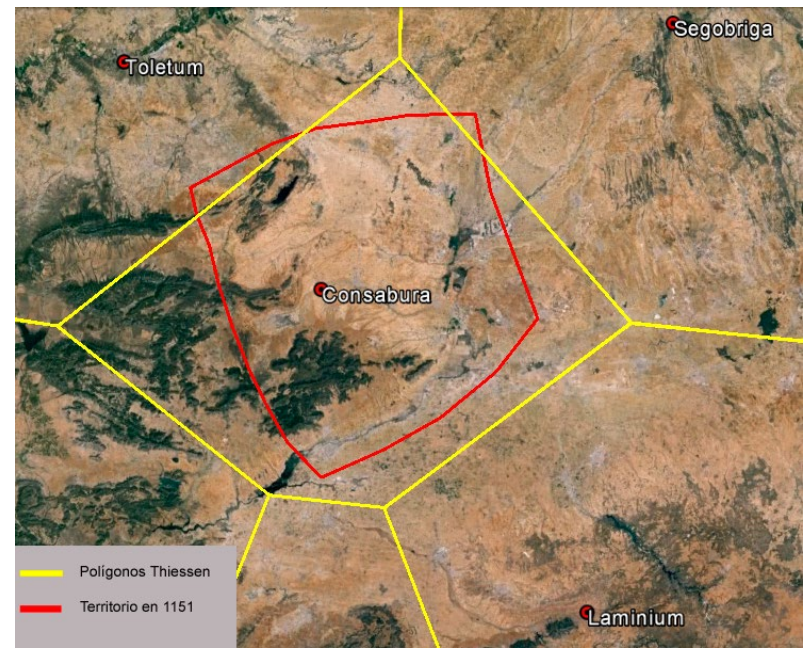

Figura 1: El territorio de Consabura. Elaboración propia a partir de Google Earth.

- Al noroeste, Sierra del Algodor y de La Rabera. Coincide aproximadamente con el punto intermedio entre Toletum y Consabura, hay un río de cierta importancia, el Algodor, y contamos además con el topónimo de Finisterre.

- Al noreste y al este: Vía 29 del Itinerario Antonino; este trazado coincide al Este aproximadamente con el curso del río Gigüela y la zona de lagunas endorreicas, una zona que cuenta con una importante densidad de yacimientos de época romana y que algunos autores han relacionado directamente con el auge de la ciudad de Consabura y sus salinas (Muñoz, 2008, 528-532 y 2011, 117-124).

- Sureste y sur: Río Gigüela.

- Suroeste y oeste: Sierra de la Calderina y Sierra del Robledo, estribaciones orientales de los Montes de Toledo9. Por otro lado, contamos con un importante camino que las crónicas medievales citan como el «Camino de Andalucía» (Arias, 2004).

Contamos con otro importante indicio sobre el probable territorium de Consabura, estos límites son muy similares a los que aparecen en la demarcación conocida más antigua de la ciudad de Consuegra: se trata del documento de donación del castillo de Consuegra por parte de Alfonso VII de Castilla al caballero Rodrigo Rodríguez, en 1151 (Ruiz Gómez, 2003, 172), donde se establecen unos límites claros: Lillo al noreste, Bogas y Mora al norte, la vía Toledo-Calatrava al oeste, la sierra de la Calderina al suroeste, el canal de Griñón y los Ojos del Guadiana al sur, y el distrito catastral de Criptana y el río Ansares al este.

9. Agradecemos enormemente al profesor de la Universidad de Alicante Juan Manuel Abascal, sus apreciaciones relacionadas con la ampliación del sector oeste del territorium de Consabura, vinculándose a las tierras del alto Bullaque. 
Este método de comparar el territorium de una ciuitas romana con la delimitación medieval de la misma ha sido aplicado en otros casos para el estudio del territorio de ciudades romanas (Curchin, 1991, 181182; Sáez, Ordóñez y García-Dils, 2010, 409).

\section{LOS FRAGMENTOS DE CERÁMICA DE BARNIZ NEGRO Y SU LOCALIZACIÓN GEOGRÁFICA}

Somos conscientes de que la denominación «campaniense» está hoy superada; es más exacto referirnos a estas cerámicas como «de barniz negro « (Principal y Ribera, 2013, 45 y 53) y, en la medida de lo posible, tratar de especificar el lugar de origen de cada fragmento para conseguir saber si estamos ante una producción «calena intermedia», «etrusca», «napolitana tardía», etc. No obstante, para la descripción de los fragmentos que presentamos a continuación, vamos a utilizar la antigua terminología de "Campanienses A, $\mathrm{B}$ y $\mathrm{C}$ » ya que no nos es posible conocer el origen exacto de la mayoría de los fragmentos que presentamos; en los casos en los que, debido a la tipología, decoración o características físicas evidentes, podamos asegurar el lugar de fabricación, lo haremos.

Empezaremos mencionando los fragmentos identificados en el oppidum del Cerro Calderico de Consuegra, para posteriormente tratar los localizados en los yacimientos de su territorium.

Los cinco fragmentos inéditos (Fig. 2), encontrados en las inmediaciones de la ladera NE del Cerro Calderico de Consuegra, son el resultado de prospecciones superficiales sin contexto arqueológico.

El fragmento de mayor tamaño de los conservados $\left(2^{\prime} 6 \times 4^{\prime} 9 \times 1{ }^{\prime} 3 \mathrm{~cm}\right)$, es un fragmento de base y pie ligeramente vuelto (Fig. 2, no 61 y fig. 3, 1), la pasta es de color beige o marrón claro, fina, dura y depurada, pero algo granulosa, ya que presenta inclusiones micáceas y calizas. Mientras que el barniz no es muy denso, de color negro, brillante, y que no cubre el fondo externo del vaso. Respecto a la decoración presenta cuatro

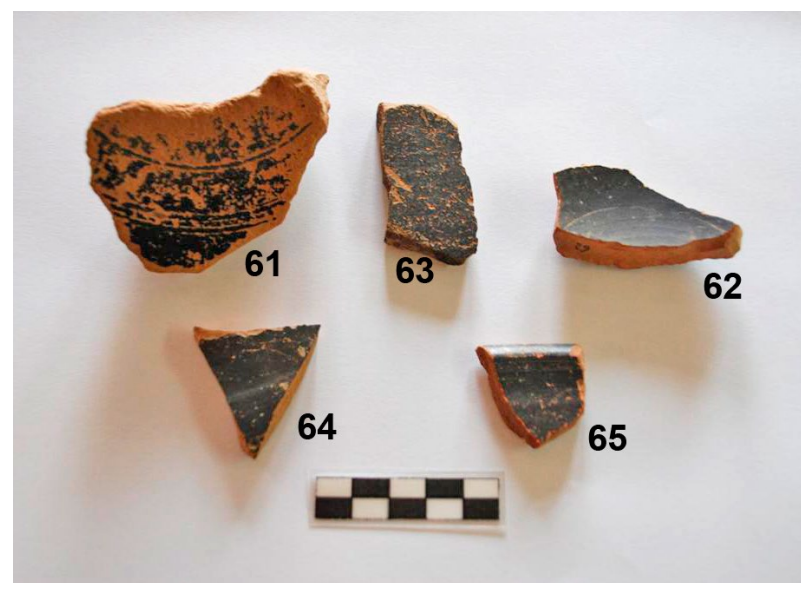

Figura 2: Fragmentos inéditos de cerámica de barniz negro en Consuegra (Toledo). Museo Municipal. círculos concéntricos incisos sobre el fondo interno, los cuales enmarcan una franja de estrías pequeñas y finas, cuyo origen se encuentra en las producciones helenísticas. La combinación de círculos y estrías es casi exclusiva de los platos Lamb. 5 y 7 , y del cuenco Lamb. 8b, aunque también cabe señalar algunos ejemplares esporádicos sobre cuencos Lamb. 1 y 8 a. Creemos que por la curvatura de su perfil y la base recta de su pie anular moldurado, es probable que se trate de la forma Lamb. 8 a/Morel F 2566, tipología asociada con el consumo de alimentos semisólidos o de líquidos de manera individual. Se trataría, pues, de un vaso polivalente. Además, por sus características físicas y formales, podemos vincularlo con producciones calenas del círculo de la Campaniense B, entre el último cuarto del s. II a.C. y finales del tercer cuarto del s. I a.C. (Principal, 2005, 55, 58; Principal y Ribera, 2013, 64).

Por otra parte, existen tres fragmentos de pared, el primero de los mismos (Fig. 2, $\mathrm{n}^{\circ} 62$ ), se diferencia de los otros por su pasta anaranjada, barniz denso tanto externo como interno, como por su perfil curvo. Pudiéndose tratar de un fragmento de cuenco o copa de tipo calena tardía (90 a.C-20 a.C.).

Los dos restantes son planos, pertenecientes a formas abiertas de platos o páteras (Fig. 2, $\mathrm{n}^{\mathrm{o}} 63 \mathrm{y} \mathrm{n}^{\circ} 64$ ), creemos que se tratarían de nuevo de producciones calenas, ya que la pasta es de arcilla calcárea de color beige claro, fina y muy bien depurada, aunque con inclusiones micáceas y calizas, a veces visibles a simple vista (fragmento $\mathrm{n}^{\mathrm{o}}$ 64-473). Por otra parte, el barniz, aplicado por inmersión, es poco denso.

Por último, destacar un fragmento de borde (Fig. 2, $\mathrm{n}^{\mathrm{o}} 65$ y Fig. 3, 2), cuya pasta es de color rojo-marrón, granulosa, con finísimas partículas de mica, duras y rugosas al tacto, mientras que su barniz negro, delgado, diluido, se desprende con facilidad en la zona de las aristas, y presenta matices metálicos. Estas características parecen relacionarlo con la fase tardía de las producciones napolitanas (ca. 100-40 a.C. Principal y Ribera, 2013, 115-116). Respecto a la forma, creemos que se relacionaría con la copa Lamb. 33 b/Morel F 2973, 2977-2978, cuyo labio exvasado presenta un bisel con angulación marcada en la parte interna del borde, que la hacen fácilmente identificable. El cálculo de su radio es de 9' $5-10 \mathrm{~cm}$, lo cual coincide con los diámetros de borde para este tipo de copas, comprendidos entre los 15 y los $20 \mathrm{~cm}$. Esta forma no suele presentar decoración, y su presencia en los yacimientos se hace más explícita a partir del s. II a.C. (Vivar, 2005, 33-34).

Como mencionábamos, todos estos fragmentos aparecieron en el sector noreste del Cerro Calderico, salvo un sexto que pertenecía a «una asita de una tapadera» (Fig. 3, 3), hallada en las excavaciones del circo romano de Consuegra, y que puede describirse como un fragmento de asas de pasta gris sin más, aunque su descubridor la catalogó como Campaniense $\mathrm{C}$ (Giles, 1971, 153); De hecho, lugares destruidos como 
consecuencia del conflicto sertoriano (78-72 a.C.), como la propia Valencia, Cabezo de Alcalá (Azaila, Teruel) o La Caridad (Caminreal, Teruel) registran la presencia en cantidades muy limitadas de Campaniense C (González, 2005, 69-70). La pieza está desaparecida, por lo que no se ha podido contrastar.

Por otra parte, y centrándonos en el territorium de la ciudad de Consabura, hemos identificado en la Carta Arqueológica de Villacañas, en el yacimiento denominado El Tomillar, un fragmento inédito de galbo o pared de una probable producción calena antigua, creemos que perteneciente a la etapa entre el 150-120 a.C., ya que presenta una pasta amarillenta clara a beige rosada, granulosa, con finísimas partículas de mica, duras y rugosas al tacto, con fisuras y vacuolas ocasionales (Principal y Ribera, 2013, 83). El barniz es negro mate, diluido, y aparece algo desprendido en las aristas ${ }^{10}$. Presenta en la cara interna líneas de torno bien marcadas (Principal y Ribera, 2013, 83). Este yacimiento presenta evidencias de una prolongada utilización durante distintos periodos. Se encuentran sobre todo restos de un asentamiento de la Edad del Hierro que tiene continuidad durante el periodo romano republicano y altoimperial. Además de materiales de estos periodos, encontramos también algunas cerámicas que podrían adscribirse a la Edad Media y a la Edad Moderna. Se trata, por tanto, de un complejo yacimiento con una dilatada ocupación en el tiempo motivada por unas condiciones favorables como son la proximidad a la Laguna Larga y a la Cañada Real Soriana, así como la disponibilidad de extensos y fértiles campos de cultivo. La ocupación más extensa e importante corresponde a la II Edad del Hierro y, sobre todo, al periodo romano. La presencia de abundante material constructivo de filiación romana, así como fragmentos de cerámica común y cerámicas típicas como la Terra Sigillata altoimperial o algunos ejemplares de cerámicas que podrían ser de tradición indígena, pone de manifiesto una cierta pujanza del asentamiento durante este periodo y además pensamos que nos encontramos ante un asentamiento de rango medio (Domingo y Magariños, 2008, 80).

En el límite noreste de nuestra zona de estudio, y próximo a la vía 29 del Itinerario de Antonino, hallamos el importante oppidum de unas $18 \mathrm{ha}$, denominado Cerro del Gollino (Corral de Almaguer, Toledo), cuya cronología se sitúa entre el s. VI-I a.C. En este yacimiento han aparecido tres platos (Fig. 4) de Campaniense B, forma Lamb. 5/Morel F 2252, 2254 y 2257-2258. Se trata de un plato de fondo llano y borde rectilíneo sin inflexión angulosa marcada en la pared externa, y con pie anular triangular. Presenta una decoración incisa de círculos concéntricos sobre fondo interno sin franja de estrías y sin marcas estampilladas de sellos. Su marco cronológico podría situarse entre

10. Fragmento siglado: 07-45-185-0056. Carta Arqueológica de Villacañas. Domingo y Magariños, 2008. Museo de Santa Cruz de Toledo.
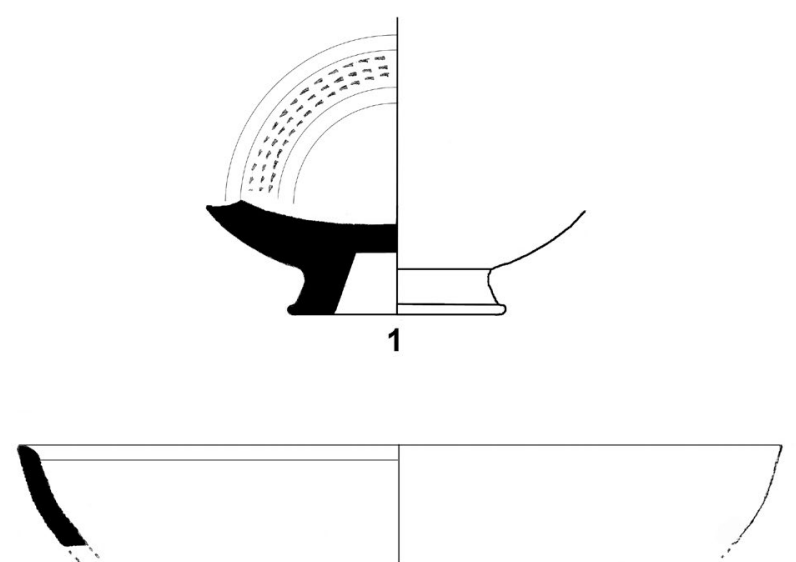

2

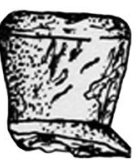

3

Figura 3: Lamb. 8a (1) y Lamb. 33b (2) procedentes del Cerro Calderico (Consuegra); Fragmento procedente del Circo Romano de Consuegra (3); Galbo de forma indeterminada de «El Tomillar», Villacañas (4).
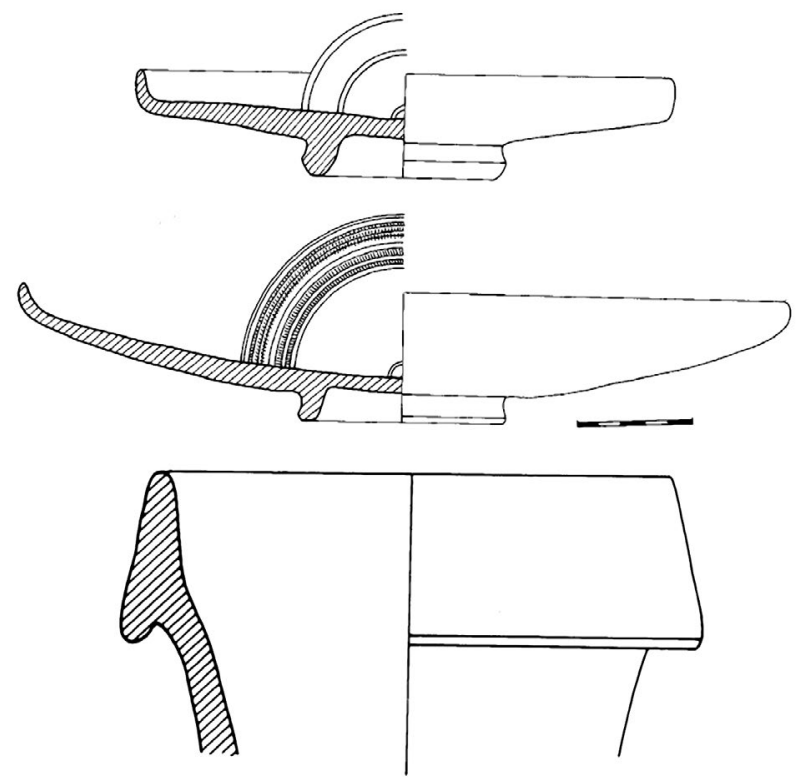

Figura 4: Materiales de cerámica Campaniense B (probablemente calena) y ánfora Dressel 1. «Cerro del Gollino» (Corral de Almaguer, Toledo). Santos, Perea y Prados, 1998, 67-68.

mediados del s. II a.C. y finales del s. I a.C. Su utilización se relacionaría con el servicio de alimentos. En el mismo yacimiento contamos con fragmentos de la forma Lamb. 1 de la serie F 2320, 2361 de Morel, se trata de un cuenco bajo, de pared-borde rectilíneo que presenta normalmente dos surcos incisos en paralelo 


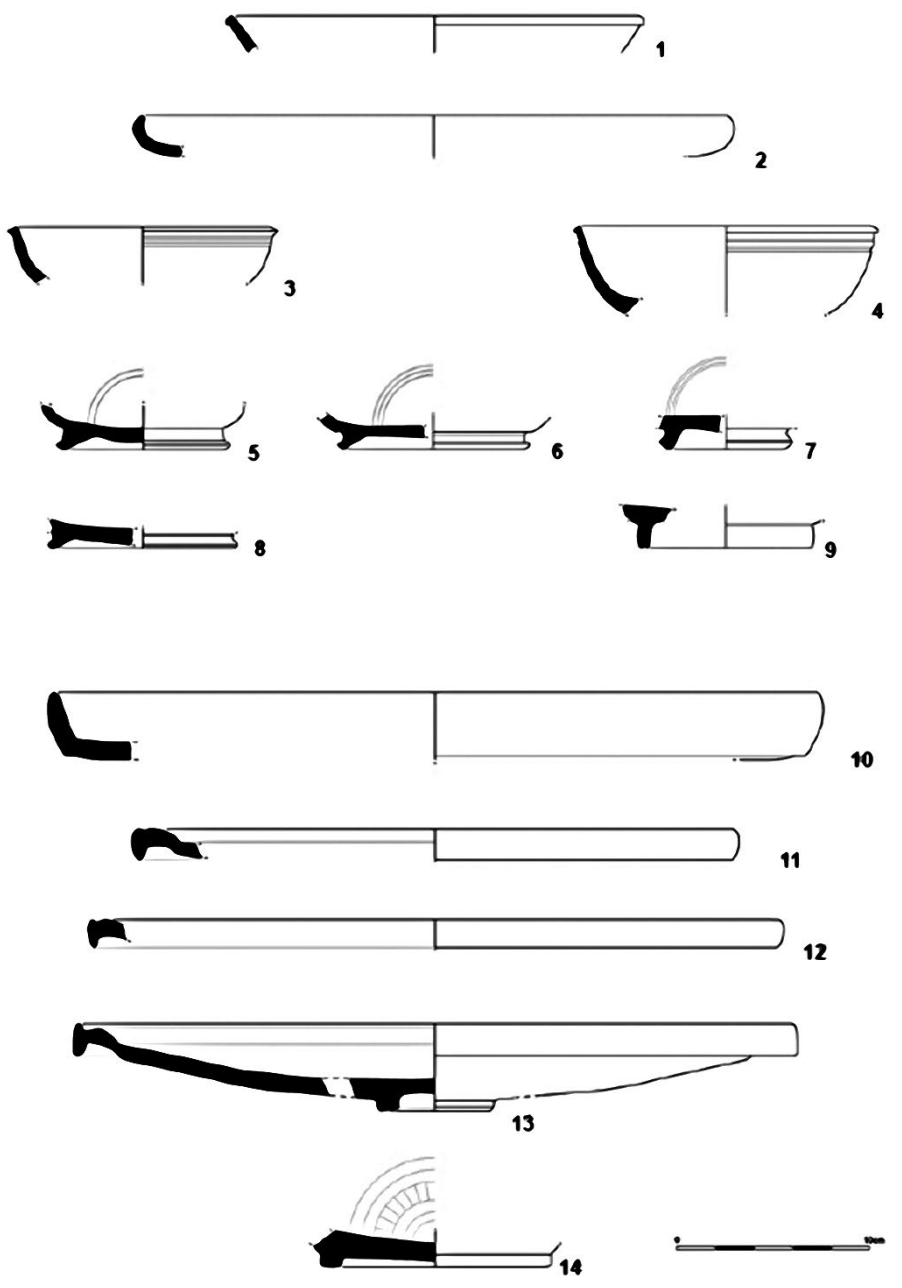

Figura 5: Cerámica de barniz negro procedente de Torre Sevilla (Alcázar de San Juan, Ciudad Real). Morín de Pablos, $2010,309$.

bajo borde externo; pie anular ligeramente exvasado, de sección triangular, sirva como ejemplo el fragmento de pie que se encontró en la base interna de la muralla (en el relleno que macizaba ambos paramentos. Santos, Perea y Prados, 1990, 314-315). Esta forma es propia de las producciones campanas medias de Cales. Su marco cronológico podría situarse entre finales del tercer cuarto del siglo II a.C. e inicios del último cuarto del I a.C. La utilización se relacionaría con el consumo de alimentos semisólidos o de líquidos de manera individual. Se trataría, pues, de un vaso polivalente (Principal, 2005, 54). La presencia de estos materiales de importación junto a otros como vasos de paredes finas, o ánforas Dressel 1, nos hablan del grado de romanización de este yacimiento, que sin embargo, se abandonaría en el s. I a.C. (Santos, Perea y Prados, 1998, 69).

Otros fragmentos son los aparecidos en el yacimiento de Pozo Sevilla (Alcázar de San Juan, Ciudad Real), excavado por el equipo de Jorge Morín de Pablos $^{11}$. Con todas las reservas que merece el caso, Pozo

11. Arqueólogo de AUDEMA S.A. responsable de varias intervenciones en la comarca que se caracterizan por su metódico
Sevilla podría englobarse entre los ejemplos de «casas-torres» destinadas a servir de apoyo a la instalación de elementos latinos o romanizados en el campo manchego. Las estructuras documentadas y la cronología de los hallazgos en un periodo tardorrepublicano avalaría, al menos de modo hipotético, la existencia de una construcción de esas características relacionada con el clima de inseguridad creado en el s. I a.C. y con la puesta en explotación del territorio circundante. En este sentido, la relación entre la estructura turriforme y el pozo nos parece muy interesante. De hecho, esta relación entre torre y pozo se explicaría por el interés en controlar uno de los escasos puntos de abastecimiento de agua (Morín de Pablos et alii, 2010, 287).

Destaca la gran cantidad de material arqueológico recuperado, con cerca de 2.500 fragmentos de cerámica; de éstos, cerca de 40 fueron identificados como

buen hacer y por la divulgación, aspectos que no abundan en nuestra zona; le agradecemos su disposición y colaboración, a la hora de elaborar este artículo, por facilitarnos la información necesaria sobre los hallazgos de cerámica de barniz negro en las intervenciones que él ha efectuado. 
de barniz negro itálico. El análisis de sus pastas y barnices permite reconocer que dos de ellos se inscriben dentro de las producciones napolitanas de Campaniense A, concretamente de la forma Lamb. 28a-b/ Morel F 2612-2614, 2641-2648, 2654 (Fig. 5, 1 y 9), y que la mayoría, con pastas beiges y barnices negros con reflejos metálicos, pertenecen a las producciones calenas del círculo de la Campaniense B. En cuanto a las formas, se encuentran atestiguadas la Lamb. 1/ Morel F 2320, 2361, Lamb. 5 / Morel F 2252, 2254, 2257-2258, Lamb. 5-7/ Morel F 2282-2284 y Lamb. 7 / Morel F 2283-2284, 2286 (Fig. 5, 2-8).

Además de éstas, están representadas en igual número las que se pueden atribuir a las Campanienses grises o lo que se empieza a denominar como Cerámica Gris Bruñida Republicana (GBR, Adroher y Caballero, 2012, 23-38), concretamente grandes platos/páteras de las formas Lamb. 7 y Lamb. 6 (Fig. 5, 10-13), y un fondo de forma indeterminada con pasta gris, y ausencia de barniz negro, que es sustituido por un fuerte bruñido de la superficie. Este último, presenta decoración radial en su parte interna, que con bastantes reservas se clasifica por sus excavadores como una producción de C, o una derivada de ésta (Fig. 5, 14). No obstante la evidente heterogeneidad al nivel de las producciones, la cronología del conjunto resulta bastante coherente. Se trata de un conjunto con asociaciones formales típicas de los contextos del segundo y del tercer cuarto del siglo I a.C, donde cabe la presencia de los dos fragmentos de Campaniense A, aparentemente de producción tardía. (Morín de Pablos et alii, 2010, 310). Desde nuestro punto de vista, estamos de acuerdo en la coherencia cronológica del material arqueológico, ya que las producciones napolitanas tardías suelen aparecer junto a una mayoría abrumadora de fragmentos de origen caleno en torno al s. II-I a.C. (Principal y Ribera,
2013, 95). Si a ello, unimos la presencia de cerámica gris bruñida republicana (GBR), la lógica cronológica y funcional del yacimiento es aún mayor, ya que estas producciones de imitación de barniz negro parecen relacionarse con el mundo militar tardorrepublicano (Adroher y Caballero, 2008, 327-328).

En lo que respecta a las ánforas, es notoria su elevada presencia y porcentaje. Dentro de un conjunto compuesto por 80 fragmentos, se pueden reconocer junto a las formas típicas del Hierro II (Pellicer B-C y D), los de vino itálico típicos del siglo I a.C., las Dressel 1.

Por otro lado, y cambiando de área geográfica, a unos $20 \mathrm{~km}$ al norte de Consuegra, tenemos constancia de la aparición de varios fragmentos cerámicos pertenecientes a las producciones del Círculo de la Campaniense B, entre los S. II-I a.C., en el citado yacimiento de Pozos de Finisterre, junto a varios bordes de ánforas de imitaciones del tipo Dressel 1, correspondientes también a este período republicano (Hernández y Morín de Pablos, 2008, 84-85).

\section{CONCLUSIONES}

En este trabajo hemos podido analizar como en la etapa de finales de la República se produjeron sustanciales cambios en la Meseta Sur, como la desaparición de algunos centros poblacionales en beneficio de otros, privilegiados por Roma. Esta nueva reestructuración del territorio de las ciudades puede rastrearse a través de las calzadas, de la ubicación de los yacimientos y de la aparición en los mismos de cerámicas de barniz negro de origen itálico o Campanienses. En particular de los tipos A (procedente de la Isla de Ischia y del Golfo de Nápoles) y B (producciones calenas y etruscas), que nos fechan con cierta exactitud los niveles en donde nos

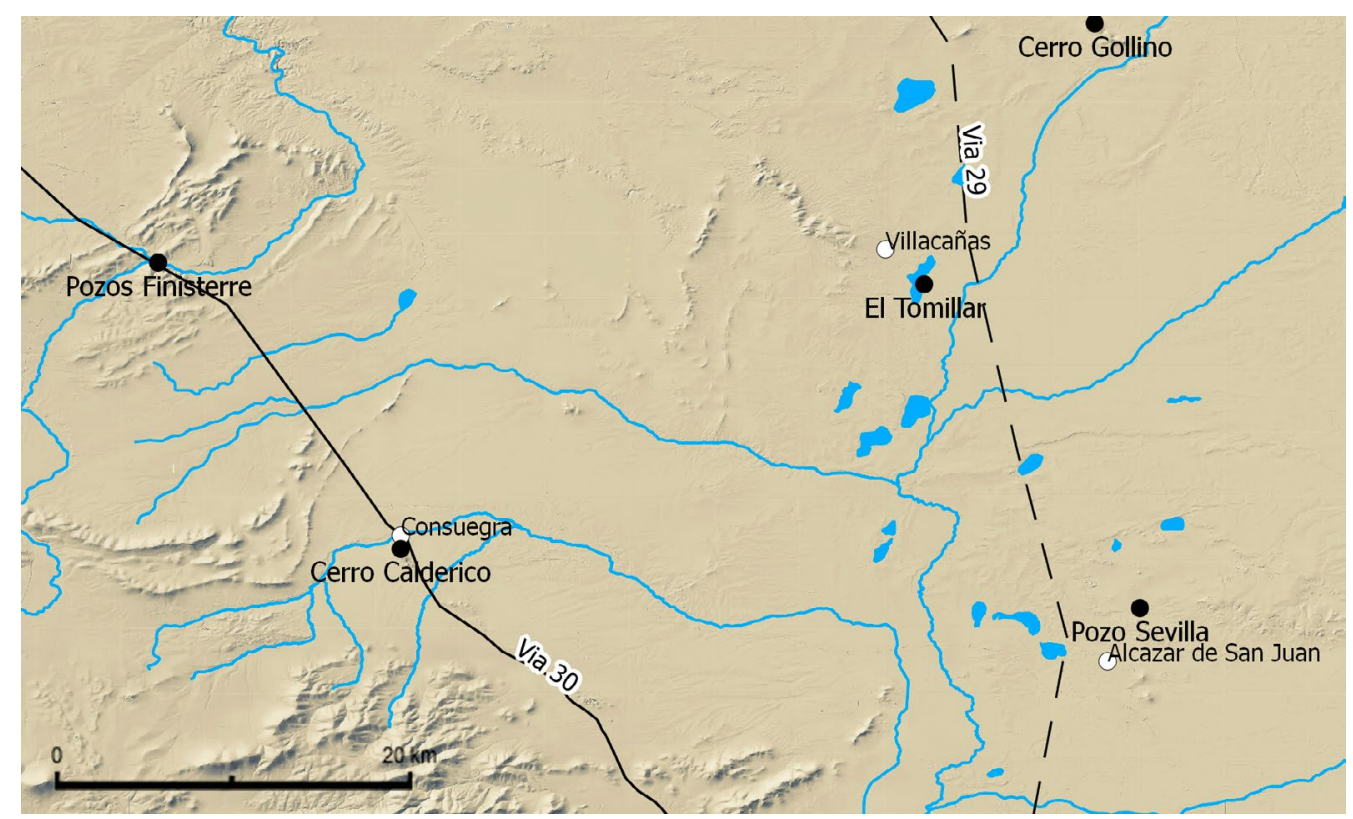

Figura 6: Yacimientos y vías de comunicación que se citan en el texto. 


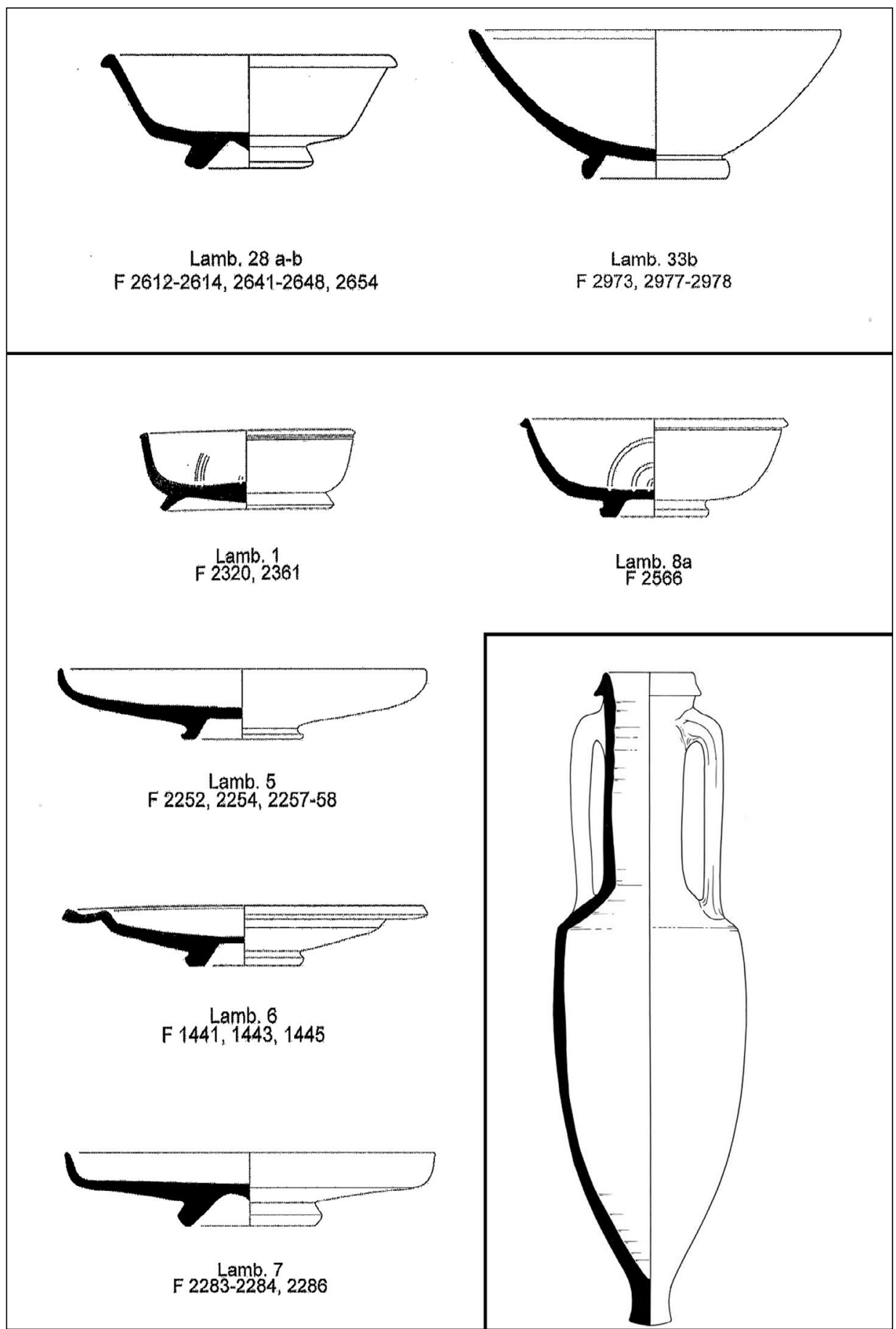

Figura 7: Vajilla de barniz negro y ánfora Dressel 1 en el territorium de Consabura.

aparecen, así como el grado de romanización de las zonas en cuestión. A esto habría que añadir todos aquellos elementos como: cascos, armas (glandes), aumento del numerario republicano (por ejemplo los denarios de la ceca Bolskan) y la aparición de los llamados «tesorillos de ocultación», etc; elementos íntimamente relacionados con los movimientos de tropas dentro de un período tan convulso como fue el de las guerras sertorianas (82-72 a.C.), cuya primera etapa transcurrió entre los valles del Tajo y del Guadiana (PS. FRONTIN. Strat. IV, 5, 19), y por tanto, en el territorium de la antigua Consabura y de sus civitates limítrofes (Toletum, Segobriga, Laminium).
Otro aspecto, a nuestro parecer muy interesante, es el de conocer las vías de penetración de estas producciones en nuestro territorio. Ante el hecho de tratarse, en la mayoría de los casos de recipientes destinados a su uso en la mesa, bien para beber, bien para servir, presentar o consumir alimentos (Pérez, 2007, 210), y vista la distribución de los materiales a lo largo de los dos grandes ejes viarios de la zona: vías 29 y 30 (Fig. 6 ), es muy probable que su introducción en la Meseta esté ligada a la presencia de tropas que participaron en el conflicto de Sertorio. Creemos que las vías de difusión nos transmiten dos centros de distribución, 


\begin{tabular}{|c|c|c|c|}
\hline Yacimiento & HALLAZGO & FORMAS & CRONOLOGIA \\
\hline 1. Cerro Calderico (Consuegra, Toledo) & $\begin{array}{c}5 \text { frag.: } 1 \text { frag. Campaniense A y } 4 \\
\text { frag. Campaniense B (calena). } 2 \text { frag. } \\
\text { de TSI }\end{array}$ & \begin{tabular}{|c|} 
Lamb. 33 b/F 2973, \\
2977-2978; Lamb. 8 a \\
/F2566 \\
\end{tabular} & S. II-I a.C. \\
\hline 2. Circo romano (Consuegra, Toledo) & 1 frag. Campaniense $\mathrm{C}$ & Indeterminada & S. I a.C. \\
\hline 3. E1 Tomillar (Villacañas, Toledo) & 1 frag. Campaniense B & Indeterminada & $\begin{array}{c}\text { Campaniense B Media/Clásica } \\
(180-100 \text { a.C. })\end{array}$ \\
\hline $\begin{array}{l}\text { 4. E1 Cerro de1 Gollino (Corral de Almaguer, } \\
\text { Toledo) }\end{array}$ & $\begin{array}{l}3 \text { platos y } 3 \text { frag. Campaniense B } \\
\text { (calena). Ánfora Dressel } 1\end{array}$ & \begin{tabular}{|c|} 
Lamb. 5/ F 2252, 2254 \\
y 2257-2258. Lamb. \\
$1 /$ F 2320, 2361 \\
\end{tabular} & $\begin{array}{c}\text { Mediados del s. Il a.C.-finales } \\
\text { del s. I a.C. }\end{array}$ \\
\hline $\begin{array}{l}\text { 5. Pozo Sevilla (A kázar de San Juan, Ciudad } \\
\text { Real) }\end{array}$ & $\begin{array}{l}40 \text { frag. en total: } 2 \text { de Campaniense } \\
\text { A. Mayoría Campaniense B. } 1 \text { frag. } \\
\text { de Campaniense C. }\end{array}$ & Lamb. 28a-b & $\begin{array}{c}\text { Segundo-Tercer cuarto del s. I } \\
\text { a.C. }\end{array}$ \\
\hline 6. Pozos de Finisterre (Consuegra, Toledo) & $\begin{array}{l}4 \text { frag. de Campaniense B. } \\
\text { Imitaciones Dressel } 1\end{array}$ & Indeterminada & S. II-I a.C \\
\hline
\end{tabular}

Figura 8: Tabla de yacimientos del territorium de Consabura con materiales cerámicos de importación de época tardorrepublicana.

una vez que estos materiales llegaron por vía marítima a suelo hispano. Así es muy probable que estos productos se distribuyeran desde el valle del Ebro y ello es interesante, porque la existencia de contactos comerciales entre el medio Ebro y el Tajo, está constatada también por la importante circulación monetaria (Abascal, 1995, 174; Azcárraga y Gamo, 2012, 143), las cecas de Bolskan, Bilbilis, Calagurris, Cascantum, Celsa, entre otras, son relativamente abundantes en nuestro territorio durante la etapa tardorrepublicana y augustea ${ }^{12}$. Consideramos que estas importaciones llegan hasta el valle del Guadiana, en el que se ubica nuestro territorio objeto de nuestro estudio. De hecho, si recordamos el mapa que sitúa los hallazgos de cerámica Campaniense (Fig. 6), observaremos que muchos de estos yacimientos se sitúan en el sector este del territorio, muy próximos a la vía 29 del Itinerario de Antonino (Per Lusitaniam ab Emerita Caesarea Augusta), que vinculaba nuestra zona con el valle del Ebro, pero también debemos de considerar otras vías de penetración de estos materiales cerámicos, que son de nuevo ratificadas por la numismática, como es el caso de las conexiones con Laminium, punto de confluencia de las principales vías del territotorium de Consabura, y por tanto, con los caminos que traerían la influencia romana desde Levante.

Incluso, y a modo de hipótesis, creemos que podemos ir más allá, es razonable suponer que desde comienzos del siglo I a.C. se diera el establecimiento progresivo de itálicos en esta zona, por supuesto no tan importante como en el valle del Guadalquivir o en la costa levantina, pero sí significativo. Un indicio en este sentido puede ser el hecho de que Consabura resistiera al ejército de Sertorio, aliándose por consiguiente con el gobierno de Roma. Esta actitud se puede comprender si se supone un sector de la población identificado con el gobierno de la $V r b s$, para la cual la causa sertoriana, apoyada por los pueblos de reciente conquista, no podía dejar de aparecer como una causa ilegítima. Una población en la que hubiera un peso importante de los colonos itálicos, evidentemente se

12. Como demuestran las colecciones numismáticas del Museo Municipal de Consuegra (Toledo). sentiría más identificada con el gobierno de la República que con la sublevación de los vecinos lusitanos instigada por Sertorio (Salinas de Frías, 2007, 62-63). Los datos, al mismo tiempo, arqueológicos parecen confirmar esta hipótesis (Santos, Perea y Prados, 1998, 68-69).

La importación de cerámicas itálicas de barniz negro (Figs. 7 y 8), evidentemente se refiere a los gustos de una minoría, de una élite de colonos y de indígenas aculturados que demandan los productos de fabricación itálica como un símbolo de prestigio social.

\author{
Juan Francisco Palencia García \\ Dpto. Historia Antigua \\ Sede Central de la UNED \\ Facultad de Geografía e Historia \\ 28040 Madrid \\ jonpalence@gmail.com \\ Diego Rodríguez López-Cano \\ Dpto. Prehistoria y Arqueología \\ Sede Central de la UNED \\ Facultad de Geografía e Historia \\ 28040 Madrid \\ coltranch@hotmail.com
}

\section{BIBLIOGRAFÍA}

ABASCAL PALAZÓN, J.M., 1995: «Excavaciones y hallazgos numismáticos de Fernando Sepúlveda en Valderreboyo (1877-1879)», Wad-Al-Hayara, 22, 151-174.

AGUIRRE, D., 1769: El Gran Priorato de San Juan de Jerusalén en Consuegra en 1769, (Reedición del I.P.I.E.T. Toledo, 1973).

ARIAS, G., 2004: Item a Laminio Toletum del Itinerario de Antonino, en www. laxxxdeantonino.es.mn

AZCÁRRAGA, S. y GAMO, E., 2012: «Cerámica de barniz negro de época romana republicana en yacimientos celtíberos y carpetanos de la provincia de Guadalajara», Lucentum, XXXI, 131-146.

BELTRÁN LLORIS, M., 1990: Guía de la Cerámica Romana, Zaragoza.

CARRASCO SERRANO, G., 2008: «La intervención romana en Castilla-La Mancha: la anexión del territorio», en G CARRASCO SERRANO (Coord.), La Romanización en el territorio de Castilla-La Mancha, 13-32, Cuenca. 
CURCHIN, L.A., 1991: Roman Spain. Conquest and Assimilation, London and N. York.

CURCHIN, L.A., 2012: «The Urban Experience in CastillaLa Mancha in the Roman Period», en G. CARRASCO SERRANO (Coord.): La ciudad romana en Castilla-La Mancha, 15-28, Cuenca.

DOMINGO, L.A. y MAGARIÑOS, J.M., 2008: Memoria, Carta Arqueológica de Villacañas, Junta de Comunidades de Castilla-La Mancha. Inédito.

FERNÁNDEZ OCHOA, C., SELDAS FERNÁNDEZ, I. y ZARZALEJOS PRIETO, M., 1990: «Entre Consabro y Laminio: aproximación a la problemática de la vía 30 del Itinerario de Antonino», Simposio sobre la red viaria en la Hispania Romana, 165-182,Zaragoza.

FERNÁNDEZ-LAYOS DE MIER, J.C., 1983: Historia de Consuegra. Tomo I: Edad Antigua, Toledo.

GILES PACHECO, F.J., 1971: «Contribuciones al estudio de la arqueología toledana. Hallazgos hispanorromanos en Consuegra», Anales Toledanos, V, 139-165.

GONZÁLEZ LÓPEZ, M.A., 2005: «La vajilla de barniz negro. Campaniense C», en M. ROCA ROUMENS y $\mathrm{M}^{\mathrm{a}}$. I. FERNÁNDEZ GARCÍA (Coords.), Introducción al estudio de la cerámica romana. Breve guía de la cerámica romana, 63-80, Málaga.

HERNÁNDEZ, M. y MORÍN DE PABLOS, J. (Coords.), 2008: Caminería romana en la provincia de Toledo. El yacimiento de Pozos de Finisterre (Consuegra, Toledo), Marq Audema, Serie Época Romana/Antigüedad Tardía 1, Madrid.

HODDER, I. y ORTON, C., 1990: Análisis espacial en arqueología, Barcelona.

INSTITUTO GEOLÓGICO Y MINERO DE ESPAÑA: http://www.igme.es/internet/default.asp

MONTERO RUIZ, I., RODRÍGUEZ MONTERO, S. y ROJAS RODRÍGUEZ-MALO, J. M. (1990): Arqueometalurgia en la provincia de Toledo, Toledo.

MORÍN DE PABLOS, J. (Ed. científico), 2013: Pozo Sevilla (Campañas 2008-10) ¿Una casa-torre en la Mancha?, MArq Audema 2013, Serie Época Romana/Antigüedad Tardía, Madrid.

MUÑOZ VILLARREAL, J.J., 2005: «Consabura de oppidum a municipio», Hispania Antiqua, 29, 97-112.

MUÑOZ VILLARREAL, J.J., 2008: «Las salinas de Consabura (Consuegra, Toledo)», en J. MANGAS y M.A. NOVILLO (Eds.), El territorio de las ciudades romanas, 527-556, Madrid.

MUÑOZ VILLARREAL, J.J., 2011: «Evolución de la ciudad y el territorio de Consabura (Consuegra). Épocas prerromana y romana», en F. DOMÍNGUEZ GÓMEZ y J. GARCÍA CANO (Coords.), Consuegra en la Historia, 75-127, Toledo.

PALENCIA GARCÍA, J.F., 2011: «Consabura: una de las ciudades romanas más desconocidas de la antigua Carpetania», en F. DOMÍNGUEZ GÓMEZ y J. GARCÍA
CANO (Coords.), Consuegra en la Historia, 129-177, Toledo.

PALENCIA GARCÍA, J.F. y RODRÍGUEZ LÓPEZ-CANO, D., 2013: «La importancia de la minería en el área de Madridejos en la gestación de una ciudad romana», Cuadernos de Historia y Cultura Popular, $\mathrm{n}^{\circ}$ 1, 58-71.

PÉREZ, J., 2007: «La cerámica de barniz negro como mercancía. Comercio y redistribución en Hispania», Actas $V$ Jornadas Internacionales de Arqueología Subacuática, 209-270, Valencia.

PRINCIPAL, J., 2005: «Las cerámicas del Círculo de la Campaniense $\mathrm{B}$ », en M. ROCA ROUMENS y $\mathrm{M}^{\mathrm{a}}$. I. FERNÁNDEZ GARCÍA, (Coords.), Introducción al estudio de la cerámica romana. Breve guía de la cerámica romana, 47-62, Málaga.

PRINCIPAL, J. y RIBERA I LACOMBA, A., 2013: «El material más apreciado por los arqueólogos. La cerámica fina. La cerámica de barniz negro», en A. RIBERA I LACOMBA(Coord.), Manual de cerámica romana. Del mundo Helenístico al Imperio Romano, 43-146, Alcalá de Henares.

RODRÍGUEZ MORALES, J., 2007: «Algunos apuntes sobre el posible trazado de las vías romanas en la Comunidad de Madrid», El Nuevo Miliario, no 4, 20-37.

RUIZ GÓMEZ, F., 2003: Los orígenes de las Órdenes Militares y la repoblación en los territorios de La Mancha (1150-1250), Madrid.

SÁEZ, P., ORDÓÑEZ, S. y GARCÍA-DILS, S., 2010: «Infraestructuras hidráulicas en el territorio de una colonia romana de la Bética: el caso de Astigi, Colonia Augusta Firma (Écija, Sevilla, España)», en L. LAGÓSTENA, J.L. CAÑIZAR y Ll. PONS (Eds.), Aqvam perdvcendam curavit: captación, uso y administración del agua en las ciudades de la Bética y el Occidente Romano, 409-438, Cádiz.

SALINAS DE FRÍAS, M., 2007: «Los carpetanos: siglos III a.C. al I a.C.», en G. CARRASCO SERRANO (Coord.), Los pueblos prerromanos en Castilla-La Mancha, 3766, Cuenca.

SANTOS, J., PEREA, A. y PRADOS, L., 1990: «Primeros resultados de las excavaciones arqueológicas en el Cerro del Gollino (Corral de Almaguer, Toledo)», Actas del Primer Congreso de Arqueología de la Provincia de Toledo, 309-325, Toledo.

SANTOS, J., PEREA, A. y PRADOS, L., 1998: «El hábitat carpetano del Cerro del Gollino», Iberia, 1, 53-72.

SCHATTNER, T. G., OVEJERO, G. y PÉREZ, J. A., 2008: "Avances sobre el territorio de Munigua», en J. MANGAS y M.A. NOVILLO (Eds.), El territorio de las ciudades romanas, 129-154, Madrid.

VIVAR LOMBARTE, G, 2005: «La cerámica Campaniense A», en M. ROCA ROUMENS y Mª I. FERNÁNDEZ GARCÍA (Coords.), Introducción al estudio de la cerámica romana. Breve guía de la cerámica romana, 23-46, Málaga. 